\title{
ON THE PARALLELIZABILITY OF THE SPHERES
}

\author{
BY R. BOTT AND J. MILNOR
}

Communicated by H. Samelson, February 13, 1958

(The following note consists of excerpts from two letters.) (Milnor to Bott; December 23, 1957.)

... Hirzebruch tells me that you have a proof of his conjecture that the Pontrjagin class $p_{k}$ of a $G L_{m}$-bundle over the sphere $S^{4 k}$ is always divisible by $(2 k-1)$ !. I wonder if you have noted the connection of this result with classical problems, such as the existence of division algebras, and the parallelizability of spheres.

According to $\mathrm{Wu}$ the Pontrjagin classes of any $G L_{m}$-bundle, reduced modulo 4 , are determined by the Stiefel-Whitney classes of the bundle. (See On the Pontrjagin classes III, Acta Math. Sinica vol. 4 (1954) in Chinese.) The proof makes use of the Pontrjagin squaring operation, together with the coefficient homomorphism $i: Z_{2} \rightarrow Z_{4}$. Although I do not know the exact formula which Wu obtains, the following special case is not hard to prove:

Lemma. If the Stiefel-Whitney classes $w_{1}, w_{2}, \cdots, w_{4 k-1}$ of $a G L_{m^{-}}$ bundle are zero then the Pontrjagin class $p_{k}$, reduced modulo 4 , is equal to $i_{*} w_{4 k}$.

For a bundle over $S^{4 k}$ this means that $w_{4 k}$ is zero if and only if $p_{k}$ is divisible by 4 . Now if you can prove that $p_{k}$ is divisible by $(2 k-1)$ ! it will follow that $w_{4 k}$ must be zero, whenever $k \geqq 3$.

TheOREM. There exists a $G L_{m}$-bundle over $S^{n}$ with $w_{n} \neq 0$ only if $n$ equals $1,2,4$ or 8 .

PRoof. Wu has shown that such a bundle can only exist if $n$ is a power of 2 . But the above remarks show that the cases $n=16,32, \ldots$ cannot occur.

COROLlaRY 1. The vector space $R^{n}$ possesses a bilinear product operation without zero divisors only for $n$ equal to $1,2,4$ or 8 .

PRoof. Given such a product operation the map $S^{n-1} \rightarrow G L_{n}$ defined by $x \rightarrow$ (left multiplication by $x$ ) gives rise to a $G L_{n}$-bundle over $S^{n}$ for which it can be shown that $w_{n} \neq 0$.

CoROllary 2. The sphere $S^{n-1}$ is parallelizable only for $n-1$ equal to 1,3 or 7 .

Proof. Given linearly independent vector fields $v_{1}(x), \cdots, v_{n-1}(x)$, 
on $S^{n-1}$, the correspondence

$$
x \rightarrow\left(x, v_{1}(x), \cdots, v_{n-1}(x)\right)
$$

carries $S^{n-1}$ into the Stiefel manifold of $n$-frames in $R^{n}$. Identifying this space with $G L_{n}$, we again obtain a $G L_{n}$-bundle over $S^{n}$ with $w_{n} \neq 0 \ldots$

(Bott to Milnor, January 6, 1958.)

... Here is what I can show:

TheOREM. Let $B=B_{U}$ be the universal base-space of the infinite unitary group. Then the image of $\pi_{2 n}(B)$ in $H_{2 n}(B)$ is divisible by precisely $(n-1)$ !.

This then refines the result of Borel-Hirzebruch that these classes are divisible by $(n-1)$ ! except for the prime 2, [3], and confirms their conjecture. Because the Pontryagin classes are in the last analysis pre-images of classes in $B_{U}$, it follows that for any $G L_{n}(R)$ bundle over $S^{4 k}, p_{k}$ is divisible by $(2 k-1) !$. This is all you needed.

The precise divisibility of $p_{k}$, for a real bundle over $S^{4 k}$, is actually given by:

$$
p_{k}=0, \begin{aligned}
& \bmod (2 k-1) !, \quad k \text { even, } \\
& \bmod (2 k-1) ! 2, \quad k \text { odd. }
\end{aligned}
$$

This is seen by considering the fibering $U / O \rightarrow B_{O} \rightarrow B_{U}$.

The theorem follows from the fact, that if $\Omega=\Omega S U$ is the loopspace on $S U$, then there exists a homotopy equivalence $f: B \rightarrow \Omega$, as was announced in [1] and is proved in [2]. By standard theory the double suspension, $S$, from $\Omega$ into $B$, defines a homomorphism $\pi_{k}(\Omega) \rightarrow \pi_{k+2}(B)$ which is bijective for dimensions $\geqq 1$.

Let $\lambda=f_{*} \circ S$. It is then clear that:

$$
\pi_{2 k}(\Omega)=\lambda^{k-1}\left(\pi_{2}(\Omega)\right) .
$$

Now in [2] the Hopf algebra $H_{*}(\Omega)$ is described. It turns out that $H_{*}(\Omega)=Z\left[\sigma_{1}, \sigma_{2}, \cdots\right], \operatorname{dim} \sigma_{i}=2 i$, the diagonal map being: $\Delta_{*} \sigma_{i}$ $=\sum \sigma_{s} \otimes \sigma_{t} ; s+t=i ; \sigma_{0}=1$. Hence the primitive subspace, $P_{*}$, is generated by elements $\left\{p_{n}\right\}, n=1,2, \cdots$, which are inductively determined by the relation:

$$
p_{n}-p_{n-1} \cdot \sigma_{1}+p_{n-2} \cdot \sigma_{2}-\cdots \pm n \sigma_{n}=0, \quad n=1,2, \cdots .
$$

Let $\lambda_{*}$ be the homomorphism corresponding to $\lambda$ in homology. It will preserve spherical classes, and annihilate decomposable elements. It therefore follows from (2) that $\lambda_{*} p_{i}= \pm i \lambda_{*} \sigma_{i}$. As the spherical classes generate $P_{*}$ (over the rationals, $S U$ is a product of odd spheres!) this 
relation implies that $\lambda_{*}^{k-1}\left(H_{2}(\Omega)\right)$ is divisible by at least $(k-1)$ !. By (1) it follows that the spherical classes in dimension $2 k$ are divisible by at least $(k-1)$ !. This is the best bound because it is not hard to see that $\lambda_{*}$ is not divisible on all of $H_{*}(\Omega)$.

An easy corollary of the theorem is that $\pi_{2 n}\left(U_{n}\right)=Z / n ! Z$. Kervaire also has decided the parallelizability question. He uses this formula as his starting point....

\section{REFERENCES}

1. R. Bott, The stable homotopy of the classical groups, Proc. Nat. Acad. Sci. U.S.A. vol. 43 (1951) pp. 933-935.

2. - The Pontryagin ring of $\Omega G$ (to be published in Michigan Math. J.).

3. A. Borel and F. Hirzebruch: Characteristic classes and homogeneous spaces (to be published in Amer. J. Math.).

UNIVERSITY OF MICHIGAN AND OXFORD UNIVERSITY 